\title{
ASSISTÊNCIA À SAÚDE E DEMANDA DOS SERVIÇOS NA ESTRATÉGIA SAÚDE DA FAMÍLIA: A VISÃO DOS ADOLESCENTES
}

\author{
Roberta Peixoto Vieira ${ }^{1}$, Maria de Fátima Antero Sousa Machado², Italla Maria Pinheiro Bezerra³, \\ Caroline Antero Machado ${ }^{4}$
}

RESUMO: Objetivou-se conhecer a percepção dos adolescentes acerca da assistência à saúde e demanda dos serviços na Estratégia Saúde da Família. A entrevista foi o instrumento de coleta dos dados, aplicada a 20 adolescentes de uma escola da rede pública municipal de Barbalha-Ceará, de junho a agosto de 2009. A organização do material foi orientada pela identificação de temas a partir das falas. Evidenciou-se que os adolescentes buscam a Unidade de Saúde da Família motivados principalmente pela doença, não se sentem como parte do serviço e expressam o desejo por atividades que conduzam a uma maior participação. Constatou-se que ainda existe a necessidade de organização do processo de trabalho das Equipes de Saúde no sentido de captar esta clientela, estimular e fortalecer o vínculo, bem como consolidar a política voltada à saúde dos adolescentes.

PALAVRAS-CHAVE: Adolescente; Atenção primária à saúde; Assistência.

\section{HEALTHCARE AND THE DEMAND FOR SERVICES IN THE FAMILY HEALTH STRATEGY: THE PERSPECTIVE OF ADOLESCENTS}

ABSTRACT: The study's objective was to study how adolescents see healthcare and the demand for services in the Family Health Strategy. Data was collected via interviews, carried out with twenty adolescents from a state school in Barbalha-Ceará, Brazil, between June and August 2009. Material was organized by themes identified in the discourse. Evidence was found that adolescents approach the Family Health Units principally when sick, that they do not feel part of the service, and that they express a desire for activities which would lead to greater participation. It was ascertained that there is still a need for organization of the Health Teams' work processes with a view to involve this clientele, as well as to consolidate policy directed at adolescent health. KEYWORDS: Adolescent; Primary health care; Care.

\section{ASISTENCIA A LA SALUD Y DEMANDA DE LOS SERVICIOS EN LA ESTRATEGIA SALUD DE LA FAMILIA: LA VISIÓN DE LOS ADOLESCENTES}

RESUMEN: El objetivo del estudio fue conocer la percepción de los adolescentes acerca de la asistencia a la salud y demanda de los servicios en la Estrategia Salud de la Familia. La entrevista fue el instrumento para recoger los datos, aplicada a 20 adolescentes de una escuela de la red pública municipal de Barbalha-Ceará, de junio a agosto de 2009. La organización del material fue orientada por la identificación de temas a partir de las hablas. Se evidenció que los adolescentes buscan la Unidad de Salud de la Familia a causa principalmente de enfermedades, no se sienten parte del servicio y expresan el deseo por actividades que conduzcan a una mayor participación. Se concluye que todavía hay la necesidad de organización del proceso de trabajo de los Equipos de Salud en el sentido de captar esta clientela, estimular y fortalecer el vínculo, así como consolidar la política designada a la salud de los adolescentes.

PALABRAS-CLAVE: Adolescente; Atención primaria a la salud; Asistencia.

${ }^{1}$ Enfermeira. Especialista em Saúde da Família. Integrante do Grupo de Pesquisa Saúde Coletiva da Universidade Regional do CaririGRUPESC/URCA.

${ }^{2}$ Enfermeira. Doutora em Enfermagem. Professora do Curso de Enfermagem da URCA. Líder do GRUPESC.

${ }^{3}$ Enfermeira. Mestre em Modelos de Decisão e Saúde. Professora do Curso de Enfermagem da URCA e Faculdade de Juazeiro do Norte. Membro do GRUPESC.

${ }^{4}$ Cirurgiã-dentista. Mestre em Saúde Coletiva. 


\section{INTRODUÇÃO}

A adolescência se configura como uma fase da vida marcada por profundas mudanças no âmbito biopsicosocial, as quais são muito rápidas e vividas muito intensamente. Por conseguinte, vem o amadurecimento, que é o objetivo desta fase marcada por duas aquisições importantes: a capacidade reprodutora e a identidade pessoal ${ }^{(1)}$.

Essa fase de vida é considerada como um período marcado pela vulnerabilidade, entendida como a capacidade do indivíduo de decidir sobre sua situação de risco, estando diretamente associada a fatores individuais, familiares, culturais, sociais, políticos, econômicos e biológicos. E alguns desses fatores podem aumentar o grau de vulnerabilidade dos adolescentes frente às diferentes situações e problemas vivenciados na adolescência ${ }^{(2)}$.

Nesse contexto, um dos alcances do conceito de vulnerabilidade é dado pelo seu potencial de ampliação sobre a compreensão dos fenômenos da saúde, considerando seus determinantes e condicionantes, assim como as ações de saúde voltadas para a prevenção e controle dos agravos ${ }^{(3)}$.

Desta forma, a reflexão deste conceito é essencial para que os profissionais de saúde identifiquem características e/ou condições para potencializar os recursos disponíveis para o enfrentamento dos determinantes do processo saúde-doença, já que a identificação de condições, características e situações de proteção e o fortalecimento dos indivíduos e grupos constituem um dos diferenciais do conceito de vulnerabilidade.

Tendo em vista a importância da promoção da saúde no contexto da saúde coletiva, faz-se necessário que sejam viabilizadas e direcionadas as ações da Estratégia Saúde da Família (ESF) que ultrapassem os ideais e valores dos modelos hegemônicos privatista e campanhista, ainda presentes nos serviços de saúde. Sendo assim, pode-se dizer que a ESF se configura atualmente como uma política de saúde pública que pode favorecer a mudança da saúde do adolescente, através da capacidade de promover assistência integral continuada baseada nos princípios do Sistema Único de Saúde (SUS).

Evidências mostram que $80 \%$ dos problemas de saúde podem ser solucionados na Atenção Básica. Contudo, sabe-se que os serviços de saúde encontram dificuldades em atender adolescentes e jovens. Estes passam pelo SUS 'invisibilizados', por não serem reconhecidos em sua especificidade etária ${ }^{(4)}$, o que acarreta uma assistência à saúde do adolescente que não considera suas particularidades e especificidades.
Para garantir a atenção integral a esse grupo populacional, é condição primordial promover a assistência compreendendo os aspectos biológicos, psicológicos e sociais da saúde do sujeito, inserido em contextos social, cultural e familiar e em um território(4).

Nessa perspectiva, com intuito de buscar melhorias no atendimento, no acolhimento e nas respostas às demandas dos adolescentes é necessário que os profissionais da equipe de Saúde da Família construam seu processo de trabalho voltado para a prática do cuidado familiar ampliado, desenvolvendo atividades grupais de educação em saúde; implementando estratégias integradas, setoriais e intersetoriais de produção de saúde; valorizando a participação e o desenvolvimento da autonomia e promovendo a participação desse grupo populacional em redes intersetoriais que lhes garanta proteção e o cumprimento de seus direitos ${ }^{(4-5)}$.

Com base na percepção do contexto da saúde do adolescente e serviços de saúde, conduziu-se este estudo a partir das seguintes perguntas norteadoras: Os adolescentes procuram os serviços de saúde? Como os adolescentes percebem seu atendimento nas Unidades de Saúde da Família? Quais as expectativas dos adolescentes diante do serviço? Para responder aos questionamentos, objetivou-se conhecer a percepção dos adolescentes acerca da assistência à saúde e demanda dos serviços na Estratégia Saúde da Família.

\section{METODOLOGIA}

Estudo descritivo qualitativo, realizado no $\mathrm{Mu}-$ nicípio de Barbalha-Ceará-Brasil, o qual possui 21 Unidades de Saúde da Família (USF) e 21 Equipes de Saúde da Família, sendo que 11 delas localizam-se na zona urbana e 10 na zona rural, além de contar com dois Núcleos de Apoio à Saúde da Família $(\mathrm{NASF})^{(6)}$.

As equipes das USF são compostas por médico, enfermeiro, técnico de enfermagem, cirurgião dentista, auxiliar de consultório dentário e agentes comunitários de saúde. As Unidades oferecem serviços de saúde da criança (puericultura), saúde da mulher (pré-natal, planejamento familiar, prevenção de câncer de colo uterino), saúde do idoso, saúde bucal, imunização, serviço ambulatorial, ações programáticas a usuários portadores de hipertensão arterial, diabetes mellitus, tuberculose e hanseníase, entre outros. Os NASF contam com equipes formadas por fisioterapeuta, pediatra, psicólogo, avaliador físico e farmacêutico, que oferecem serviços na Atenção Básica e na média complexidade ${ }^{(6)}$. 
Foi escolhido como locus deste estudo uma escola da rede pública, opção que se deu pelo fato desta escola atender adolescentes de diversas localidades do município, favorecendo assim a investigação de realidades diferentes no que se refere aos serviços de saúde na Atenção Básica, e por proporcionar o contato com adolescentes que frequentavam as USF, bem como aqueles que não o faziam.

Os critérios de inclusão dos sujeitos no estudo foram: estar na faixa etária de 10 a 19 anos; estar regularmente matriculado e frequentando a escola; apresentar o termo de consentimento assinado; e quando menor de 18 anos, apresentar o termo de consentimento assinado pelos pais ou responsável. Considerou-se como recorte para o grupo de informantes da pesquisa, adolescentes na faixa etária definida no estudo de uma sala de aula escolhida aleatoriamente.

Os dados foram coletados no período de junho a agosto de 2009, por meio da entrevista semiestruturada, cujo instrumento abordou aspectos referentes à relação dos adolescentes com o serviço de saúde. Houve a entrega do Termo de Consentimento Livre e Esclarecido aos 32 alunos que se encontravam em sala, havendo retorno de 20 destes, que se constituíram a amostra do estudo.

Foram seguidos os critérios da Resolução 196/96 $6^{(8)}$ que regulamenta pesquisa com seres humanos, sendo a participação dos sujeitos voluntária. Ainda foram assegurados a confidencialidade e o sigilo dos respondentes, informando-os de que sua participação ou não na mesma não acarretaria qualquer benefício indevido, nem prejuízo de maneira alguma. O estudo recebeu autorização do Comitê de Ética em Pesquisa da Faculdade de Medicina de Juazeiro, Juazeiro do Norte-CE, sob o parecer n. 0343/2009.

O material empírico foi organizado seguindo os passos da técnica de Análise de Conteúdo ${ }^{(7)}$, sendo a análise consubstanciada por fontes secundárias da literatura pertinentes à temática: artigos de periódicos nacionais e internacionais e produções recentes sobre a atenção à saúde do adolescente, processo de trabalho em saúde e promoção da saúde voltada para este grupo populacional.

\section{RESULTADOS}

Considerando a faixa etária de 10 a 19 anos, estabelecida na pesquisa para participação dos informantes, dos 20 alunos entrevistados, 13 são do sexo feminino e 7 do masculino, na faixa etária entre 13 a 17 anos. Dentre os adolescentes, 12 eram da zona urbana e 8 da zona rural. O olhar deste grupo acerca da assistência prestada pelos profissionais das USF está expresso nas categorias que se seguem.

\section{Adolescentes e serviços de saúde}

A procura dos adolescentes pelo serviço de saúde, que ainda tem sido motivada pela doença e seus fatores associados, fica evidente nas falas desses sujeitos:

Eu só vou mais quando eu tô doente ou quando eu preciso de algum exame [...]. (A2-15 anos)

Eu já fui no PSF, quando eu fui pro dentista. E também fui prá uma consulta, prá pegar remédio lá (A4-13 anos).

\section{Percepção dos adolescentes sobre as ações da Equipe de Saúde da Família}

Quando indagados se a USF do seu bairro possui alguma ação para os adolescentes, poucos indicaram a distribuição de recursos contraceptivos (preservativo e pílula anticoncepcional) e os demais não reconheceram nenhuma atividade direcionada a este público alvo:

Minha prima, que é da minha idade, vai pro posto pegar camisinha, pilula [...] mas, às vezes, nem tem. (A19-17 anos)

Eu não sei se tem alguma coisa no Posto só prá adolescente não. Mas eu sei que pra idosos tem.(A4-13 anos)

Os adolescentes afirmaram também que a falta de atividades para sua faixa etária, ou a desinformação a respeito de tais atividades, age como um fator para que os mesmos não frequentem as USF:

Lá não tem palestra não. Nunca me chamaram prá nada. (A7-13anos)

Eu acho que os adolescentes não vão pro Posto porque não tem nada prá gente lá. Só quando a gente tá doente! (A20-16 anos)

Os adolescentes também revelaram surpresa ao serem indagados sobre a importância dos mesmos para o serviço de saúde:

Não me acho importante pro PSF não. Só vou lá quando eu tenho algum problema. (A8-15anos)

Acho que prá você ser importante prá alguma coisa 
ou prá alguém, eles tem que precisar de você, e lá eles não precisam de mim. (A10- 17 anos)

\section{Expectativas dos adolescentes diante do serviço}

Foi enfatizada, pelos adolescentes, a necessidade de atividades que promovessem a prevenção dos agravos comuns à adolescência e proporcionassem informação:

Ultimamente os adolescentes estão sem se prevenir, sem querer se prevenir, e ninguém ajuda. (A2-15 anos)

Acho que eles deviam falar mais, ter mais palestras, prá prevenir a gravidez e as drogas [...]. Isso pode ser feito na escola e no PSF [...]. Não ia mudar as pessoas, mas algumas dúvidas a gente precisa tirar. Às vezes, os pais têm vergonha de responder a gente, e a gente tem vergonha de perguntar e, às vezes, eles não sabem responder [...]. (A9-16anos)

\section{DISCUSSÃO}

O serviço da Atenção Básica ainda é visto apenas como um campo de práticas médico-assistencialistas. Observa-se que os adolescentes pouco utilizam o serviço de saúde e quando o fazem a assistência é centrada apenas na doença, através de consultas médicas e odontológicas, marcação de exames e entrega de medicamentos, o que se contrapõe ao modelo de organização da assistência proposto pela Estratégia Saúde da Família (ESF).

A mudança do enfoque da saúde é uma das linhas norteadoras da ESF, através da substituição do modelo tradicional de assistência voltado à cura das doenças por um modelo no qual a família interage nas diversas situações de saúde-doença. Para que a família possa ser cuidada de modo global, faz-se necessário que o cuidado aconteça antes que as situações de doença apareçam ou se agravem ${ }^{(9)}$.

Nesse processo, os adolescentes, se bem conduzidos e apropriados da importância do novo modelo, podem agir no processo de consolidação das ações da ESF através de sua participação ativa e mudança consciente de hábitos de vida, possibilitando melhoria da qualidade de vida de suas famílias e comunidade e, consequentemente, realizando a promoção da saúde no nível local.

Outros estudos também trazem essa realidade, onde os pacientes procuram o serviço público de saúde em busca de solução e alívio para o sofrimento ${ }^{(10)}$, ou, a maior parte dos usuários procura os serviços de saúde para diagnóstico e tratamento ${ }^{(11)}$. Nesse sentido, o profissional acaba direcionando suas ações para indivíduos que procuram os serviços de saúde por alguma possível patologia ${ }^{(12)}$.

Contudo, esse direcionamento de ações ainda com características de modelos anteriores aos da ESF, não é apenas decorrente da incompetência ou da falta de habilidade profissional, mas efeito da falta de reorientação dos programas voltados para os adolescentes que ainda não estão sendo implementados de forma efetiva pela política pública, o que acaba se limitando a situações de riscos, não considerando a particularidades e necessidades deste segmento populacional.

Em concordância com estas ideias, estudo revelou que as dificuldades da assistência ao público adolescente, além daquelas inerentes ao próprio sistema de saúde (recursos econômicos escassos, infraestrutura deficiente, escassez de programas de capacitação profissional, etc), decorrem de outras esferas da sociedade como, por exemplo, da educação e da cultura ${ }^{(13)}$.

Por vezes, os adolescentes relataram não saber da existência de atividades para sua faixa etária na Unidade de Saúde específica, observando-se que os mesmos desconhecem o que é preconizado pelo Ministério da Saúde para a Atenção Integral à Saúde do Adolescente, pois não conseguem identificar quais atividades poderiam ser desenvolvidas pelos profissionais da Unidade para atender suas necessidades.

Merece, pois, destacar que as condições de trabalho a que os enfermeiros das Unidades de Saúde estão submetidos são precárias, faltam materiais educativos, estrutura física inadequada para a assistência e para as atividades educativas; associados a esses fatores estão os inúmeros programas que eles devem desenvolver dentro da Unidade de Saúde, o que impossibilita o desempenho pleno voltado aos adolescentes ${ }^{(14)}$.

Por outro lado, o trabalho em saúde deve incorporar mais tecnologias leves que se materializam em práticas relacionais, como, por exemplo, acolhimento e vínculo. Essas tecnologias deveriam estar à disposição dos profissionais e poderiam ser utilizadas com mais propriedade para garantir um maior envolvimento e adesão dos adolescentes às práticas de prevenção e promoção da saúde ${ }^{(15)}$.

Pode-se perceber que o acesso dos adolescentes ao serviço de saúde ainda necessita ser facilitado, tendo em vista a necessidade de discussão das questões que permeiam essa fase da vida, faltando, no contexto investigado, o vínculo com a equipe e ações mais específicas para esse grupo. 
Ainda foi apreendido, a partir dos depoimentos, que os adolescentes não se sentem valorizados, justificando sua presença nos serviços de saúde, não por serem importantes a estes, mas pela necessidade de resolução de algum problema específico.

Esta realidade pode indicar uma falha nos serviços que não implementam ações voltadas aos adolescentes e, consequentemente, esses enfrentam problemas que decorrem da insuficiência de políticas públicas que lhes assegurem acesso à educação e saúde de qualidade ${ }^{(16)}$.

Nesse contexto, é necessário que as políticas visem, acima de tudo, a valorização dos adolescentes por meio da formação de grupos, viabilizando ações que estimulem os profissionais de saúde a implementar estratégias no sentido de favorecer o desenvolvimento pessoal e social deste grupo, contribuindo para o emponderamento e o vínculo profissional de saúde/adolescentes.

A especificidade na atenção ao adolescente se apresenta como desafio, podendo-se citar como obstáculos a serem superados pelos profissionais a necessidade de adequação da linguagem e da forma de atuação, a fim de se alcançar um nível de compreensão dos vários segmentos que constituem esta população ${ }^{(17)}$. Mas, nesse processo, deve-se considerar a forma como os adolescentes enxergam os profissionais e os serviços de saúde, e suas reais necessidades.

Ao se abordar as necessidades dos adolescentes diante do serviço foram apontadas a carência de ações de prevenção e promoção da saúde - tanto nas Unidades de Saúde da Família quanto nas escolas - que proporcionassem a discussão sobre temas e questionamentos presentes no cotidiano dos adolescentes e que, muitas vezes, acabam sem respostas.

Diante desta realidade, ações de intervenção e promoção à saúde do adolescente devem ser estabelecidas e, nessa proposta, devem-se considerar os fatores sociais, político-institucionais e pessoais como determinantes do sucesso dessas ações. A partir disso, podem-se identificar questões que aumentam o grau de vulnerabilidade dos adolescentes frente aos riscos, tais como: questões de gênero cruzadas com raça/etnia e classe social; condições de vida e saúde; acesso ou não à informação; acesso à educação formal, aos serviços de saúde, ao desenvolvimento vocacional e às oportunidades de trabalho, entre outras questões ${ }^{(2)}$.

Entretanto, para alcance dessa intervenção e promoção da saúde, a educação em saúde torna-se uma ferramenta de fundamental relevância por trabalhar a atenção integral à saúde do adolescente. Através do uso da comunicação, a educação em saúde busca conferir às pessoas conhecimentos e habilidades para que estas possam fazer escolhas sobre sua saúde, despertando a consciência crítica, reconhecendo os fatores que influenciam a saúde e encorajando-as a fazer algo para mudar o status $q u o^{(9)}$.

Compreende-se que a perspectiva de trabalho voltada à proteção e promoção da qualidade de vida para a adolescência envolve uma dimensão integradora entre múltiplos setores, tanto nos aspectos macroestruturais (as políticas), como na articulação interna para mobilização das intervenções ${ }^{(18)}$.

Alguns estudos evidenciaram o quanto é necessário rever as práticas educativas a serem realizadas com adolescentes e a frequência com que devem ser feitas; além disso, é imprescindível a participação dos educadores e familiares neste processo, já que este grupo etário ainda tem buscado informações com amigos, que nem sempre são corretas. Isto revela que tanto o setor da saúde, como o da educação, não estão dando conta da integralidade à saúde dos adolescentes ${ }^{(19)}$.

Nesse sentido, embora os serviços de saúde sejam muito importantes para assegurar a atenção das pessoas e das populações, o estado sanitário das comunidades não depende apenas deles. Muitas ações de promoção e de proteção de saúde são realizadas por outras organizações que não integram o setor, destacando-se as ações educativas realizadas no ambiente escolar, consideradas fundamentais para a promoção da saúde ${ }^{(20)}$.

Construir espaços de diálogo entre adolescentes, professores, profissionais de saúde e as famílias é, comprovadamente, um importante dispositivo para construir resposta social com vistas à superação das relações de vulnerabilidade às DST, à infecção pelo HIV e à Aids, assim como à gravidez precoce. Para tanto, as ações desenvolvidas devem ir além da dimensão cognitiva, levando em conta aspectos subjetivos, questões relativas às identidades e às praticas afetivas e sexuais no contexto das relações humanas, da cultura e dos direitos humanos ${ }^{(21)}$.

Contudo, vale ressaltar que a formação inadequada dos profissionais de saúde é uma das problemáticas centrais, que fragiliza a eficácia de se trabalhar a educação em saúde na lógica da promoção requerida pela ESF. Ainda se observam discursos permeados por uma educação voltada para as doenças e para a tentativa de mudança de comportamento dos indivíduos, com relação vertical e impositiva. Por esta razão, reitera-se a relevância da educação permanente e da reestruturação dos cursos de graduação, de modo a aproximar a práxis da educação em saúde da realidade social ${ }^{(22)}$. 


\section{CONSIDERAÇÕES FINAIS}

No presente estudo, identificou-se que a busca dos adolescentes pelo serviço de saúde ainda tem sido movida pela doença e seus fatores associados, fato que ressalta que o modelo médico assistencialista ainda se encontra como obstáculo à consolidação da atenção integral proposta pela ESF. Os adolescentes não reconhecem a USF como um campo de outras atividades que não sejam as consultas, solicitação de exames e entrega de medicamentos, além de não conhecerem os profissionais da Equipe Saúde da Família, vista a falta de políticas públicas para que isso ocorra.

A necessidade percebida pelos adolescentes para a promoção da saúde se manifesta pelo desejo dos mesmos de participar de atividades que promovam a prevenção dos agravos comuns à adolescência e de informação, ações que poderiam ser implementadas a partir de um bom acolhimento por parte dos profissionais, uma comunicação eficaz e interação com este público.

Portanto, ainda existe a necessidade de organização do processo de trabalho das Equipes de Saúde da Família, no sentido de captar esta clientela, estimular e fortalecer o vínculo entre equipe e os referidos usuários. Também há a necessidade de consolidação da política voltada à saúde dos adolescentes, centrada em atividades de promoção à saúde que propiciem o protagonismo juvenil.

\section{REFERÊNCIAS}

1. Girondi JBR, Nothaft SCS, Mallmann FMB. A metodologia problematizadora utilizada pelo enfermeiro na educação sexual de adolescentes. Cogitare Enferm. 2006;11(2):161-5.

2. Ministério da Saúde (BR). Secretaria de Atenção à Saúde. Área de Saúde do Adolescente e do Jovem. Marco legal: saúde, um direito de adolescentes. Brasília: Ministério da Saúde; 2005.

3. Sanchez AIM, Bertlozzi MA. Pode o conceito de vulnerabilidade apoiar a construção do conhecimento em Saúde Coletiva? Ciênc Saúde Coletiva. 2007;12(2):319-24.

4. Ministério da Saúde (BR). Secretaria de Atenção em Saúde. Departamento de Ações Programáticas Estratégicas. Diretrizes nacionais para a atenção integral à saúde de adolescentes e jovens na promoção, proteção e recuperação da saúde. Brasília; 2010.

5. Alves CA, Brandão ER. Vulnerabilidades no uso de métodos contraceptivos entre adolescentes e jovens: interseções entre políticas públicas e atenção à saúde. Ciênc Saúde Coletiva. 2009;14(2):661-70.

6. Ministério da Saúde. (BR) Secretaria de atenção à saúde. Estabelecimento de Saúde do Município: BARBALHA. Brasília, 2010. [acesso em 05 jul 2010]. Disponível em: http://cnes.datasus.gov.br/Lista_Es_Municipio.asp?V Estado $=23 \&$ VCodMunicipio $=230190 \&$ NomeEstado $=$ CEARA.

7. Bardin L. Análise de conteúdo. $20^{\mathrm{a}}$ ed. Lisboa: Edições 70; 2009 .

8. Ministério da Saúde(BR). Conselho Nacional de Saúde. Diretrizes e normas regulamentadoras de pesquisas envolvendo seres humanos. Resolução 196, de 10 de outubro de 1996. Brasília; 1996.

9. Machado MFAS. Compreensão das mudanças comportamentais dos usuários no PSF por meio da participação habilitadora [tese]. Fortaleza (CE): Universidade Federal do Ceará; 2007.

10. World Health Organization. Mental health and brain disorders. 2001. [acesso em 05 jul 2010]. Disponível: http://www.who.int/en/

11. Reiners AAO, Azevedo RCS, Ricci HÁ, Souza TG. Adesão e reações de usuários ao tratamento da malária: implicações para a educação em saúde. Texto e Contexto Enferm. 2010; 19(3):536-44.

12. Souza LM, Wegner W, Gorini MIPO. Educação em saúde: uma estratégia de cuidado do cuidador leigo. Rev Latino-Am. Enfermagem. 2007;15(2):337-4.

13. Nogueira MJ, Modena CM, Schall VT. Políticas públicas voltadas para adolescentes nas unidades básicas de saúde no município de Belo Horizonte/MG: uma análise sob a perspectiva dos profissionais de saúde. Rev APS. 2010;13(3):338-45.

14. Oliveira TC, Carvalho LP, Silva MA. O enfermeiro na atenção à saúde sexual e reprodutiva dos adolescentes. Rev Bras Enferm. 2008;61(3):306-11.

15. Souza ECF, Vilar RLA, Rocha NSPD, Rocha PM. Acesso e acolhimento na atenção básica: uma análise da percepção dos usuários profissionais de saúde. Cad Saúde Pública. 2008;24 Suppl 1:S100-10.

16. Horta NC, Lage AMD, Sena R.R. Produção científica sobre políticas públicas direcionadas para jovens. Rev Enferm. 2009;17(4):538-43. 
17. Ministério da Saúde (BR). Secretaria de Atenção à Saúde. Departamento de Ações Programáticas Estratégicas. Saúde do adolescente: competências e habilidades. Brasília; 2008.

18. Costa COM, Bigras M. Mecanismos pessoais e coletivos de proteção e promoção da qualidade de vida para a infância e adolescência. Ciênc Saúde Coletiva. 2007;12(5):1101-9.

19. Cunha ABO, Vieira-da-Silva LM. Acessibilidade aos serviços de saúde em um município do Estado da Bahia, Brasil, em gestão plena do sistema. Cad Saúde Pública. 2010;26(4):725-37.

20. Paim JS. O que é SUS. Rio de Janeiro: FIOCRUZ; 2009.

21. Ministério da Saúde (BR). Secretaria de Atenção à Saúde. Departamento de Ações Programáticas Estratégicas. Marco teórico e referencial: saúde sexual e saúde reprodutiva e adolescentes e jovens. Brasília; 2006.

22. Bensen CB, Netto MS, Da Ros MA, Silva FW, Silva CG, Pires MF. A Estratégia Saúde da Família como objeto de educação em saúde. Saúde Soc. 2007;16(1):57-68. 